\title{
Chern Numbers for Spin Models of Transition Metal Nanomagnets
}

\author{
C.M. Canali ${ }^{1}$ A. Cehovin ${ }^{2}$ and A.H. MacDonald ${ }^{3}$ \\ ${ }^{1}$ Department of Technology, Kalmar University, 39182 Kalmar, Sweden \\ ${ }^{2}$ Division of Solid State Theory, Department of Physics, Lund University, SE-223 62 Lund, Sweden and \\ ${ }^{3}$ Department of Physics, University of Texas at Austin, Austin TX 78712
}

(Dated: July 1, 2018)

\begin{abstract}
We argue that ferromagnetic transition metal nanoparticles with fewer than approximately 100 atoms can be described by an effective Hamiltonian with a single giant spin degree of freedom. The total spin $S$ of the effective Hamiltonian is specified by a Berry curvature Chern number that characterizes the topologically non-trivial dependence of a nanoparticle's many-electron wavefunction on magnetization orientation. The Berry curvatures and associated Chern numbers have a complex dependence on spin-orbit coupling in the nanoparticle and influence the semiclassical Landau-Liftshitz equations that describe magnetization orientation dynamics.
\end{abstract}

Both molecular nanomagnets [1, 2, 3] and ferromagnetic transition metal clusters [4, 5, 6] have been actively studied over the past decade. Interest in molecular nanomagnets has been due primarily to their position near the borderline between quantum and classical behaviors 3, 7, 8]. For ferromagnetic transition metal nanoparticles, on the other hand, interest has been spurred mainly by classical physics issues relevant to information storage 9, 10, 11 and by the interplay between collective and quasiparticle degrees of freedom 12, 13]. The present work is motivated by the observation that the low energy physics of small transition metal clusters can be described by an effective Hamiltonian with a single giant spin degree of freedom, like that of a molecular magnet. A transition metal nanoparticle will behave like a molecular magnet when the energy scale associated with its collective magnetization orientation, the anisotropy energy, does not exceed the smallest energy scale associated with its quasiparticle degrees-of-freedom, the single-particle level spacing $\delta$. When bulk density-ofstates and anisotropy energy values are used to estimate the particle-size at which this condition is satisfied, the cubic transition metal ferromagnets $\mathrm{Fe}$ and $\mathrm{Ni}$ are predicted to act like molecular magnets when the number of atoms $N_{A}$ is smaller than $\sim 1000$, while Co is predicted to act like a molecular magnet for $N_{A}$ smaller than $\sim 100$. (See Table 【). It is remarkable that an extensive $\left(\propto N_{A}\right)$ energy scale, like the total anisotropy energy, is smaller than a microscopic energy scale $\left(\propto N_{A}^{-1}\right)$, like the level spacing, at a relatively large particle number. This surprising property arises from a combination of relatively weak spin-orbit coupling in the 3d transition-metal series and the itinerant character of transition metal ferromagnetism. The magnetocrystalline anisotropy energy in these systems in the bulk is five to six orders of magnitude smaller than the magnetic condensation energy. Even accounting for the substantially larger anisotropy energy per volume expected in typically shaped nanoparticles, it seems clear that the total width of the anisotropy energy landscape will often be smaller than the level spacing for $N_{A}$ smaller than $\sim 100$. The physics that controls the Hamiltonian and, most centrally, the total spin of these malleable molecular magnets is the subject of this Letter.
TABLE I: $\delta N_{A}$ is the bulk mean-level spacing times the number of atoms. $E_{B}$ is the bulk coherent rotation anisotropy energy barrier (For cubic systems $E_{B} /$ vol is one third of magnetic anisotropy constant $K_{1}$, whereas in the uniaxial case it is equal to the magnetic anisotropy constant.) $N_{A}^{\star}$ is the number of atoms at which $\delta$ and $E_{B}$ become equal.

\begin{tabular}{ccccc}
\hline \hline & $\delta N_{A}(\mathrm{meV})^{a}$ & $E_{B} / \mathrm{vol}\left(\mathrm{MJ} / \mathrm{m}^{3}\right)^{b}$ & $E_{B} / N_{A}(\mathrm{meV})$ & $N_{A}^{\star}$ \\
\hline $\mathrm{Fe}$ & 695 & 0.016 & .00118 & 767 \\
$\mathrm{Co}$ & 581 & 0.53 & .0367 & 126 \\
$\mathrm{Ni}$ & 495 & 0.002 & .000114 & 2080 \\
\hline \hline
\end{tabular}

${ }^{a}$ From Ref. 15

${ }^{b}$ Bulk value at room temperature from Ref. 16 .

In the absence of spin-orbit and dipole-dipole interactions, the collective physics of a transition metal nanoparticle is easily understood [14]. Quasiparticle states, calculated for a particular magnetization orientation (using the spin-density-functional theory recipe for example) have definite spin-orientations. The many-particle ground state has a total spin quantum number $S=$ $\left(N_{a}-N_{o}\right) / 2$, where $N_{a}$ and $N_{o}$ are respectively the number of quasiparticle states whose individual spins are aligned with and opposed to the total spin. The $2 S+1$ fold ground-state degeneracy is lifted by an arbitrarily weak external magnetic field, selecting a ground state with magnetic moment $2 S \mu_{B}$. This simplicity dissolves into a complex muddle when spin-orbit interactions are included. Individual quasiparticle states no-longer have definite spin orientations. It is not immediately obvious how to derive an effective Hamiltonian which describes the low-energy collective physics, or even how to determine the dimension of the quantum Hilbert space for the coherent magnetization degree of freedom. We address this issue here using a path integral approach. We find that the total spin $S$ of the molecular magnet is specified by a Chern number defined by the dependence of the many-particle wavefunction phase on magnetization orientation, and propose a procedure for extracting a quantum Hamiltonian from the dependence of energy and Berry curvature on orientation. Our analysis is based on 
an approximate imaginary-time quantum action with a single magnetization-orientation degree of freedom, $\hat{n}(\tau)$ :

$$
\mathcal{S}_{\mathrm{coh}}[\hat{n}] \equiv \int d \tau\left[\left\langle\Psi[\hat{n}] \mid \vec{\nabla}_{\hat{n}} \Psi[\hat{n}]\right\rangle \cdot \frac{\partial \hat{n}}{\partial \tau}+E[\hat{n}]\right] .
$$

For simple model Hamiltonians in which the interaction terms can be represented exactly by auxiliary field function integrals 17., actions of this form can be derived fully microscopically [18]. For the realistic description of real nanoparticles, however, this action is approximate and should be constructed by using the family of spin-densityfunctional theory constrained Kohn-Sham states with net magnetization orientation $\hat{n}$ for an approximate identity resolution at energy scales below the single-particle level spacing. In the absence of spin-orbit interactions, spin-density-functional theory describes the ground state in terms of Kohn-Sham quasiparticles that experience a scalar potential and an exchange-correlation effective magnetic field [19], both of which have a complex dependence on spatial coordinate. The orientational degree of freedom we have in mind for the action is the direction $\hat{n}$ in spin-space of the spin-density-functional theory exchange-correlation effective field 20, or its spatial average if low-energy states have non-collinear magnetization. In Eq.[1] $|\Psi[\hat{n}]\rangle$ is the Kohn-Sham singleSlater determinant state defined by this orientation, with spin-orbit terms included in the Hamiltonian and $E[\hat{n}]$ is the density-functional-theory energy. The first term in this action is a Berry phase contribution 21 whose role in quantizing small-amplitude magnetic orientation fluctuations (spin-waves) of bulk transition metal ferromagnets has been successfully exploited by Niu et.al. 22, 23, 24], and other authors [25]. When spin-orbit interactions are included, both Berry phase and energy function $E[\hat{n}]$ terms will have a non-trivial dependence on orientation [18, 26]. We expect that quantitatively accurate actions can be constructed in this way for completely specified nanoparticles; in the rest of this paper we discuss some qualitative properties using a tight-binding model [27.

It is useful to express the Berry phase in a gauge invariant form [21, 28]. During its imaginary-time evolution, the unit vector $\hat{n}$ traces a closed trajectory on the unit sphere. By Stokes' theorem we can rewrite the closed line integral and express the Berry phase in terms of a surface integral over the area enclosed by the path

$\mathcal{S}_{\text {Berry }}[\hat{n}]=\oint d \hat{n} \cdot\left\langle\Psi \mid \vec{\nabla}_{\hat{n}} \Psi\right\rangle=\int_{\text {area }} \vec{\nabla}_{\hat{n}} \times\left\langle\Psi \mid \vec{\nabla}_{\hat{n}} \Psi\right\rangle \cdot \hat{n} d a$.

The Berry curvature

$$
\mathcal{C}[\hat{n}] \equiv i \vec{\nabla}_{\hat{n}} \times\left\langle\Psi[\hat{n}] \mid \vec{\nabla}_{\hat{n}} \Psi[\hat{n}]\right\rangle \cdot \hat{n},
$$

is gauge invariant and the Chern number $S$, defined as the average of the curvature over the unit sphere, is required to be half of an integer 29]. The Chern number appears below as the total spin in the effective model for the nanoparticle, hence the notation chosen above.
To discuss the Chern numbers, it is useful to start from the case of no spin-orbit coupling, for which $S=$ $\left(N_{a}-N_{o}\right) / 2$. To see this, consider the Kohn-Sham singleSlater determinant many-particle state which describes magnetization in direction $\hat{n}$ :

$$
|\Psi[\hat{n}]\rangle=\prod_{i}\left[u(\hat{n}) c_{i \uparrow}^{\dagger}+v(\hat{n}) c_{i \downarrow}^{\dagger}\right] \prod_{j}\left[-v^{\star}(\hat{n}) c_{j \uparrow}^{\dagger}+u(\hat{n}) c_{j \downarrow}^{\dagger}\right]|0\rangle,
$$

where $c_{\sigma}^{\dagger}$ 's are electron creation operators defined with the arbitrary polar angle of the coordinate system used to measure magnetization orientations as the spin-quantization axis, and $i$ and $j$ are orbital indices running respectively over $N_{a}$ majority-spin orbitals and $N_{o}$ minority-spin orbitals. We have made the gauge choice $u(\hat{n})=\cos (\theta / 2), v(\hat{n})=e^{-i \varphi} \sin (\theta / 2)$ for the $S=1 / 2$ quasiparticle coherent states, where $\theta$ and $\varphi$ are the polar and azimuthal angles used to specify $\hat{n}(\theta, \varphi)$. Given this state, the Berry connection 21, 28], $\vec{A}[\hat{n}] \equiv i\left\langle\Psi[\hat{n}] \mid \vec{\nabla}_{\hat{n}} \Psi[\hat{n}]\right\rangle$, is easily calculated

$$
\begin{array}{r}
\vec{A}[\hat{n}]=i \sum_{i}\left[u \frac{\partial u}{\partial \hat{n}}+v^{\star} \frac{\partial v}{\partial \hat{n}}\right]+i \sum_{j}\left[v \frac{\partial v^{\star}}{\partial \hat{n}}+u \frac{\partial u}{\partial \hat{n}}\right] \\
=\left(\sum_{i} 1-\sum_{j} 1\right) \frac{1-\cos (\theta)}{2 \sin (\theta)} \hat{\varphi}=\frac{N_{a}-N_{o}}{2} \frac{1-\cos (\theta)}{\sin (\theta)} \hat{\varphi},
\end{array}
$$

It follows that the Berry curvature $\mathcal{C}[\hat{n}]=\vec{\nabla}_{\hat{n}} \times \vec{A}[\hat{n}]$ is constant and equal to

$$
\mathcal{C}=\frac{N_{a}-N_{o}}{2} .
$$

In this case each majority-spin Kohn-Sham quasiparticle contributes $1 / 2$ to the Chern number while each minority-spin quasiparticle contributes $-1 / 2$.

When the spin-orbit interaction is present, the Berry curvature is no longer a constant, but the Chern number $S$ must still be half-integer. The total Chern number $S$ of the nanoparticle can still be considered as the sum over contributions from each quasiparticle state 22]. Individual quasiparticle Chern number properties are familiar from related studies, especially in connection with the topological interpretation of the quantized Hall conductance in a two-dimensional electron gas 29, 30, 31, 32. They can change only when quasiparticle level degeneracies occur. In nanoparticles without special symmetries, level degeneracies will occur at discrete values of $\hat{n}$, and at isolated values of any microscopic Hamiltonian parameter. When two levels intersect and then come apart again as a parameter is varied, then their individual Chern numbers may not be conserved but their sum is conserved 31]. As we now show, spin-orbit interactions lead to surprisingly ubiquitous and large deviations of quasiparticle Chern number values from their $\pm 1 / 2$ values in the absence of spin-orbit coupling.

In Fig. 1 we plot the variation of quasiparticle Chern numbers for representative groups of contiguous levels of 


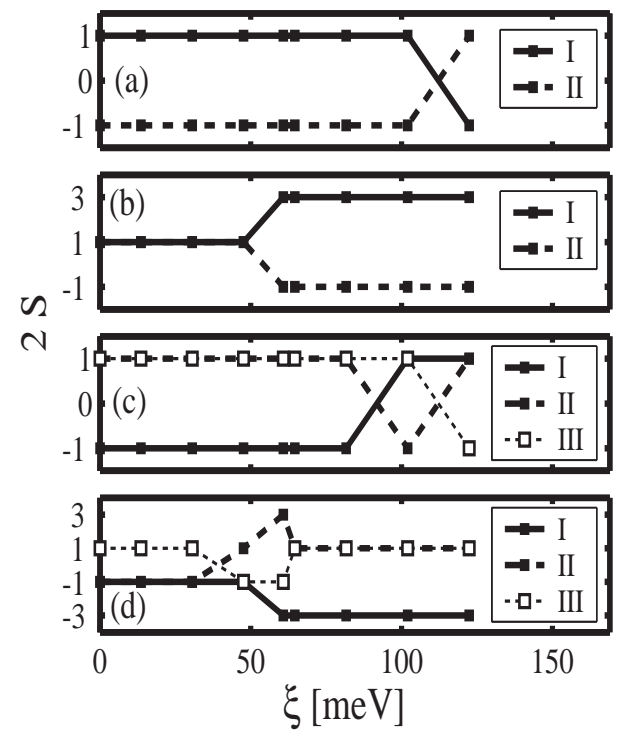

FIG. 1: Chern numbers of quasiparticle energy levels of a 25-atom Cobalt nanoparticle, as a function of the spin-orbit coupling strength $\xi$. The nanoparticle is modeled by a tightbinding Hamiltonian [27]. In each panel I, II, (and sometimes III) label two (or three) contiguous orbitals whose energies are ordered in ascending order. Deviations of individual $S$ from their zero spin-orbit coupling values $\pm 1 / 2$ occur when two levels cross. Note that the sum of the two Chern numbers is conserved at crossing.

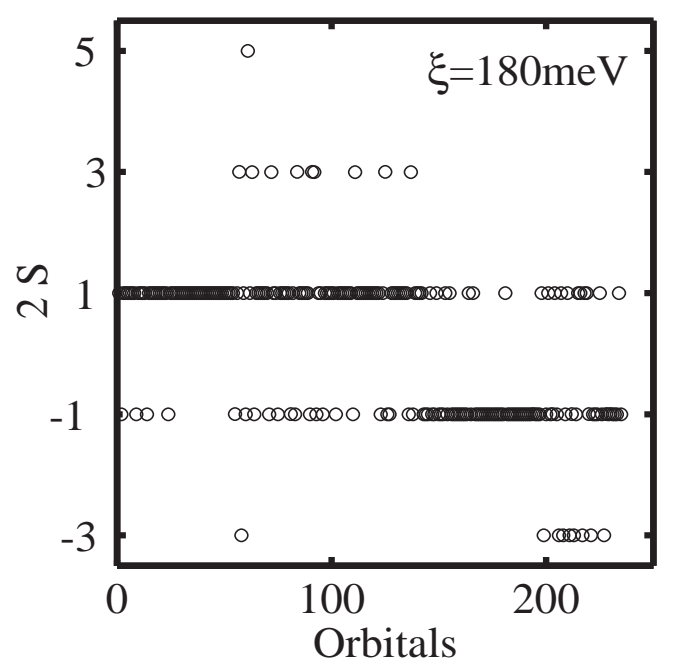

FIG. 2: Chern numbers of all the individual quasiparticle energy levels of a 25-atom nanoparticle for a given value of $\xi$. As a result of repeated level crossings, several of these numbers differ strongly from $\pm 1 / 2$.

a nanoparticle modeled by a tight-binding model [27], as a function of the spin-orbit coupling strength $\xi$. Typically we find that individual Chern numbers of pairs of levels undergoing level crossing experience a change of \pm 1 , with their sum remaining unchanged. These changes can be thought of as representing changes in the orbital

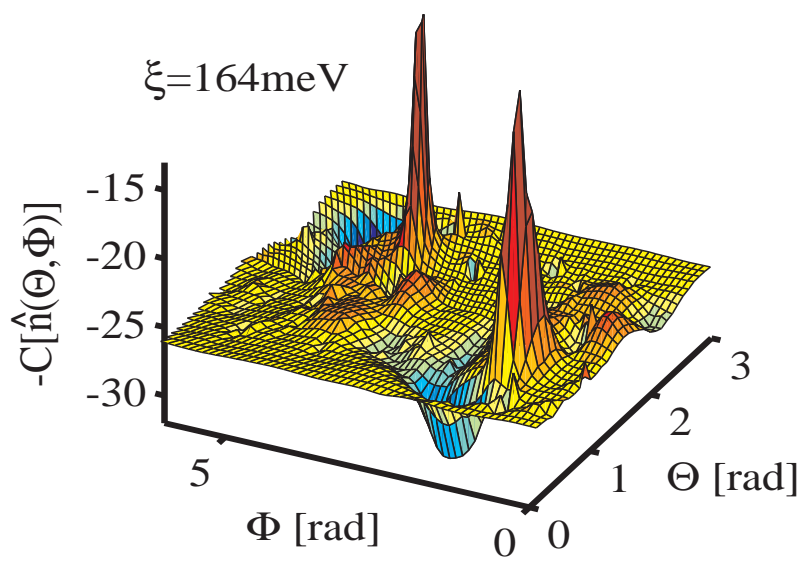

FIG. 3: Planar projection of the total (negative) Berry curvature $-\mathcal{C}[\hat{n}]$ for a 25 -atom nanoparticle. The strength of the spin-orbit coupling $\xi=165 \mathrm{meV}$ is chosen in proximity to a value at which the Fermi level and the first unoccupied level cross. Level crossing occurs for a given $\hat{n}$ and its opposite $-\hat{n}$. In correspondence of these two values, $-\mathcal{C}[\hat{n}]$ has large peaks.

contribution to the effective angular momentum of individual orbitals. Note that level crossings always occur in pairs, for a given value of $\hat{n}$ and its opposite $-\hat{n}[33]$. As a result of repeated level crossing, some of the individual Chern numbers end up with values very different from $\pm 1 / 2$ as shown in Fig. [2] where we plot all individual quasiparticle Chern numbers for a given $\xi$. If a level crossing occurs between the Fermi level and the first unoccupied level, the total Chern number will change from the original zero spin-orbit value $\left(N_{a}-N_{o}\right) / 2$. In the vicinity of such a level crossing, the Berry curvature $\mathcal{C}[\hat{n}]$ will deviate strongly from its average value as a function of $[\hat{n}]$. An example of this is shown in Fig. 3] where we plot $\mathcal{C}[\hat{n}]$ at a value of $\xi$ for which the Fermi level and the first unoccupied level are almost degenerate in two directions of $\hat{n}$ and the Berry curvature has sharp peaks.

Assuming only that the Berry curvature is positivedefinite, our low-energy effective action can be mapped to that of a quantum spin Hamiltonian by making a change of variables that transforms the Berry curvature to a constant. The Hamiltonian representation is easier to use for explicit calculations of collective tunneling amplitudes, non-linear response to external electromagnetic fields and other relevant properties. To be explicit, we change variables from $u=\cos (\theta)$ and $\varphi$ to $u^{\prime}$ and $\varphi^{\prime}$ defined by

$$
\begin{aligned}
\phi^{\prime} & =\frac{2 \pi \int_{0}^{\phi} d \varphi^{\prime \prime} \mathcal{C}\left(u, \varphi^{\prime \prime}\right)}{\int_{0}^{2 \pi} d \varphi^{\prime \prime} \mathcal{C}\left(u, \varphi^{\prime \prime}\right)} \\
u^{\prime} & =-1+\frac{1}{2 \pi S} \int_{-1}^{u} d u^{\prime \prime} \int_{0}^{2 \pi} d \varphi^{\prime \prime} \mathcal{C}\left(u^{\prime \prime}, \varphi^{\prime \prime}\right)
\end{aligned}
$$

With this change of variables $\mathcal{C}(u, \varphi) d u d \varphi=S d u^{\prime} d \varphi^{\prime}$ 
and the real-time action for a path can be written

$\mathcal{S}_{\mathrm{spin}}\left[\hat{n}^{\prime}\right]=\int_{0}^{t} d t^{\prime}\left(\vec{A} \cdot d \hat{n}^{\prime} / d t^{\prime}-\frac{S}{\mathcal{C}\left[\hat{n}\left(\hat{n}^{\prime}\left(t^{\prime}\right)\right)\right]} E\left[\hat{n}\left(\hat{n}^{\prime}\left(t^{\prime}\right)\right)\right]\right)$,

where $\vec{A}=S\left(1-\cos \left(\theta^{\prime}\right)\right) / \sin \left(\theta^{\prime}\right) \hat{\phi}^{\prime}$. This is the quantum action for a total spin quantum number $S$ with the classical Hamiltonian 28] specified by the second term of the integrand, i.e.

$$
H\left[\hat{n}^{\prime}\left(t^{\prime}\right)\right] \equiv\left\langle S, \hat{n}^{\prime}\left(t^{\prime}\right)|\mathcal{H}| S, \hat{n}^{\prime}\left(t^{\prime}\right)\right\rangle \equiv \frac{S E\left[\hat{n}\left(\hat{n}^{\prime}\left(t^{\prime}\right)\right)\right]}{\mathcal{C}\left[\hat{n}\left(\hat{n}^{\prime}\left(t^{\prime}\right)\right)\right]}
$$

where $\mathcal{H}$ is the quantum Hamiltonian of the spin system and $\left|S, \hat{n}^{\prime}\left(t^{\prime}\right)\right\rangle$ is a spin- $S$ coherent state parametrized by the unit vector $\hat{n}^{\prime}\left(t^{\prime}\right)$. Given the energy and Berry curvature functions, this quantum Hamiltonian can always be explicitly constructed. Applications of this procedure will be presented elsewhere.

A non-constant Berry curvature affects the LandauLifshitz equations describing the precession motion of the nanoparticle magnetic moments. These equations are equivalent to the Euler-Lagrange equations of motion derived from the real-time action $\mathcal{S}_{\text {spin }}\left[\hat{n}^{\prime}\right]$ in the semiclassical (large $S$ ) approximation:

$$
\dot{\hat{n}}_{\mathrm{cl}}\left(t^{\prime}\right)=\hat{n}_{\mathrm{cl}}\left(t^{\prime}\right) \times\left.\frac{\partial\left(H\left[\hat{n}^{\prime}\left(t^{\prime}\right)\right] / S\right)}{\partial \hat{n}^{\prime}\left(t^{\prime}\right)}\right|_{\hat{n}_{\mathrm{cl}}},
$$

where $\hat{n}_{\mathrm{cl}}\left(t^{\prime}\right)$ is determined by the saddle-point equations $\left.\frac{\delta \mathcal{S}_{\mathrm{spin}}\left[\hat{n}^{\prime}\left(t^{\prime}\right)\right]}{\delta \hat{n}^{\prime}\left(t^{\prime}\right)}\right|_{\hat{n}_{\mathrm{cl}}}=0$. Using the expression of $H$ in Eq. 11 we can see that a non-constant Berry curvature modifies the precession rate of the magnetic moment fluctuations. This effect is particularly important when the Fermi level and the first unoccupied state are close to a degeneracy point for some value of $\hat{n}$, since as shown in Fig [3] $\mathcal{C}[\hat{n}]$ can then deviate considerably from the Chern number $S$.

In conclusion, we have derived an effective spin Hamiltonian with a single giant spin degree of freedom that describes the low-energy physics of a small metallic nanomagnet. The dimension of the Hilbert space of the effective Hamiltonian is given by a Berry curvature Chern number which has a non-trivial dependence on spin-orbit coupling strength and nanoparticle details. We would like to thank G. Canright and W. Wersdorfer for helpful conversations. This work was supported in part by the Swedish Research Council under Grant No:621-20012357 , by the faculty of natural sciences of Kalmar University, and by the National Science Foundation under Grants DMR 0115947 and DMR 0210383.
[1] S. Sessoli, D. Gatteschi, A. Caneschi, and M. A. Novak, Nature 383, 141 (1993).

[2] J. R. Friedman, M. P. Sarachik, and J. Tejada, Phys. Rev. Lett. 76(20), 3830 (1996).

[3] W. Wernsdorfer, Adv. Chem. Phys. 118, 99 (2002).

[4] I. M. L. Billas, A. Châtelain, and W. A. de Heer, Science 265, 1682 (1994).

[5] M. Lederman, S. Shultz, and M. Ozachi, Phys. Rev. Lett. 73(14), 1986 (1994).

[6] W. Wernsdorfer, E. B. Orozco, K. Hasselbach, A. Benoit, N. Demoncy, A. Loiseau, and H. P. D. Maillya, Phys. Rev. Lett. 78(9), 1791 (1997).

[7] L. Thomas, F. Lionti, R. Ballou, D. Gatteschi, S. Sessoli, and B. Barbara, Nature 383, 145 (1996).

[8] L. Gunther and B. Barbara, eds., Quantum Tunneling of Magnetization, QTM94 (Kluwer, Dordrecht, 1995).

[9] S. A. Majetich and Y. Jin, Science 284(5413), 470 (1999).

[10] S. Sun, C. B. Murray, D. Weller, L. Folks, and A. Moser, Science 287(5460), 1989 (2000).

[11] M. Jamet, W. Wernsdorfer, C. Thirion, D. Mailly, V. Dupuis, P. Mélinon, and A. Péres, Phys. Rev. Lett. 86(20), 4676 (2001).

[12] S. Guéron, M. M. Deshmukh, E. B. Myers, and D. C. Ralph, Phys. Rev. Lett. 83(20), 4148 (1999).

[13] M. M. Deshmukh, S. Kleff, S. Guéron, E. Bonnet, A. N. Pasupathy, J. von Delft, and D. C. Ralph, Phys. Rev. Lett. 87(22), 226801 (2001).

[14] C. M. Canali and A. H. MacDonald, Phys. Rev. Lett.
85(26), 5623 (2000).

[15] D. A. Papaconstantopoulos, Handbook of the Band Structure of Elemental Solids (Plenum, New York, 1986).

[16] R. Skomski and J. M. D. Coey, Permanent Magnetism (Institute of Physics, Bristol, 1999).

[17] E. Fradkin, Field Theories of Condensed Matter Systems (Addison-Wesley, Redwood City, 1991).

[18] C. M. Canali, A. Cehovin, and A. H. MacDonald, Collective excitations in ferromagnetic metal nanoparticles (2002), unpublished.

[19] V. P. Andropov, M. I. Katsnelson, B. N. Harmon, M. van Schilfgaarde, and D. Kusnezov, Phys. Rev. B 54(2), 1019 (1996).

[20] M. Uhl, L. M. Sandratski, and J. Kuber, Phys. Rev. B 54(20), 291 (1994).

[21] R. Resta, J. Phys.: Condens. Matter 12, 107 (2000).

[22] Q. Niu and L. Kleinman, Phys. Rev. Lett 80(10), 2205 (1998).

[23] Q. Niu, X. Wang, L. Kleinman, W. M. Liu, D. M. C. Nicholson, and G. M. Stocks, Phys. Rev. Lett 83(1), 207 (1999).

[24] D. M. Bylander, Q. Niu, and L. Kleinman, Phys. Rev. B 61(18), 11875 (2000).

[25] R. Gebauer and S. Baroni, Phys. Rev. B 61(10), 6459 (2000).

[26] A. H. MacDonald and C. M. Canali, Solid State Comm. 119, 253 (2001).

[27] A. Cehovin, C. M. Canali, and A. H. MacDonald, Phys. 
Rev. B 66(10), 94430 (2002).

[28] A. Auerbach, Interacting electrons and quantum magnetism (Springer-Verlag, New York, 1994).

[29] B. Simon, Phys. Rev. Lett. 51(24), 2167 (1983).

[30] D. J. Thouless, M. K. M. P. Nightingale, and M. den Nijs, Phys. Rev. Lett. 49(6), 405 (1982).

[31] J. E. Avron, R. Seiler, and B. Simon, Phys. Rev. Lett.
51(1), 51 (1983).

[32] M. Kohmoto, Ann. of Phys. 160, 343 (1984).

[33] This is due to the degeneracy of the system with respect to $\hat{n} \rightarrow-\hat{n}$, which is rooted in the time reversal symmetry of the system in absence of an external field. 